\title{
External clinical validation of prone and supine CT colonography registration
}

\author{
Holger R. Roth ${ }^{1}$, Darren J. Boone ${ }^{2}$, Steve Halligan ${ }^{2}$, Thomas E. Hampshire ${ }^{1}$, \\ Jamie R. McClelland ${ }^{1}$, Mingxing $\mathrm{Hu}^{1}$, Shonit Punwani ${ }^{2}$, and Stuart Taylor ${ }^{2}$ \\ David J. Hawkes ${ }^{1}$ \\ 1 Centre for Medical Image Computing, University College London, UK \\ 2 Centre for Medical Imaging, Department of Specialist Radiology, University College \\ Hospital, London, UK
}

\begin{abstract}
This paper provides an external validation of a prone-supine registration algorithm for CT colonography (CTC). A validation sample of 49 patient cases with 66 polyps ( 6 to $30 \mathrm{~mm}$ ) was selected from a publicly available, anonymized CTC archive. To enhance generalizability, no case was excluded due to poor preparation or inadequate distension. Corresponding prone and supine polyp coordinates were recorded and the endoluminal surfaces registered: a Markov Random Field technique was used to find feature matches between prone/supine acquisitions and following mapping of the endoluminal surface to a cylinder, dense surface correspondence was achieved via cylindrical non-rigid registration. The polyp registration error was determined and a subjective assessment of registration made for $2 \mathrm{D}$ slice-based and $3 \mathrm{D}$ endoluminal data display using a pre-specified scoring system. Results were compared to using "normalized distance along the colon centerline" (NDACC) which approximates to the method currently employed to match colonic positions using proprietary CT colonography interpretation software. Registration was possible in all 49 cases. Overall mean 3D polyp registration error was significantly smaller with $19.9 \mathrm{~mm}$ in comparison to $27.7 \mathrm{~mm}$ using NDACC ( $\mathrm{p}=0.001) .82 .7 \%$ of polyp matches were defined as "successful" in comparison to $37.1 \%$ using NDACC according to the pre-specified criteria. Similarly, using $2 \mathrm{D}$ visualization, $62.1 \%$ registrations were "successful" and only $22.7 \%$ using NDACC. Full surface-based prone-to-supine registration can successfully map the location of a polyp identified on one acquisition to the corresponding endoluminal surface in the opposing acquisition, greatly facilitating polyp matching and aiding interpretation. Our method compares favorably to using NDACC.
\end{abstract}

Keywords: CT colonography, image registration

\section{Introduction}

CT colonography interpretation is difficult and time consuming. Fecal residue can simulate or obscure polyps, leading to both false positive and false negative diagnoses. To compensate for this, it is normal practice to obtain CT data with 
the patient both prone and supine to redistribute fecal residue and colonic gas; fecal residue tends to move, while fixed mural pathology does not. Matching corresponding endoluminal locations between prone and supine acquisitions is the cornerstone of competent interpretation [1]. Unfortunately, the colon often undergoes significant deformation during repositioning [2] which complicates the interpretative task and can induce reader error.

Software algorithms have attempted to facilitate matching of corresponding locations between prone and supine acquisitions: matching of distances along centerlines enables navigation to approximate endoluminal locations in both datasets $[3,4,5,6,7]$. The most straight-forward method (and the only technique available in commercial workstations at present) being the "normalized distance along the colon centerline" (NDACC) [8]. While centerline matching studies have shown promising results $[3,4,5,6,7]$, they tend to report registration error in only one dimension. However, it can be argued that centerline-based methods are inherently limited because the interpretation task requires readers to find a specific point on the endoluminal surface rather than along the centerline. Moreover, many readers favor using multiplanar reformats rather than endoluminal 3D displays, further limiting the utility of centerline methods in routine daily practice.

Alternative methods that aim to register the full colonic surfaces have been proposed $[9,10,11,12]$. However, such studies often report the selection criteria incompletely $[3,4,5,13,12,10]$ such that the transferability of results into clinical practice remains uncertain. For example, studies routinely use optimally prepared CT colonography datasets yet in reality, approximately $50 \%$ of cases are "poorly prepared" [14]. Likewise, validation should use data from centers that have not contributed to algorithm development (external validation) [15], to ensure previous exposure to the test data does not bias result.

We have developed software that establishes full spatial correspondence between the prone and supine endoluminal surfaces. We aim to externally validate this registration algorithm using $\mathrm{CT}$ colonography data generalizable to clinical practice and compare the results to those obtained using NDACC.

\section{Prone to supine registration}

The registration method has been described in detail previously [16]. Initialization is provided by robust matches of haustral folds between both views [17]. The haustral folds are segmented on the colon surface using a graph cut method applied to a surface curvature-based metric. Using a Markov Random Field based on similarity of folds and additional neighborhood information, fold matches are then estimated between both views. Only inverse-consistent matches were used, i.e. the same match is obtained supine-to-prone and prone-to-supine. Secondly, the entire endoluminal surface is mapped to a cylinder utilizing a conformal mapping based on Ricci flow [18]. The original surface curvature information is preserved during this step. Surface correspondence is then achieved using a nonrigid, cylindrical version of the well-known B-spline registration [19]. Registration 
is driven by local shape measurements, i.e. shape index $(S I)$ computed on the colon surface. This measure has also shown to be useful in computer-aided detection (CAD) for CT colonography [20]. The sum-of-squared differences (SSD) of these $S I$ measures are used to drive the cylindrical registration. In case of insufficient colonic distension, any locally collapsed region can be ignored during this cylindrical registration when computing the similarity measure. This is important as collapse can commonly occur in clinical practice. We arbitrarily denote the prone image as target and supine image as source when computing the registration. After convergence of the algorithm, any point on the endoluminal surface can be mapped between both corresponding acquisitions in either direction (prone-to-supine and supine-to-prone) by inverting the result $[16,17]$. Figure 1 illustrates the principle of this registration method.

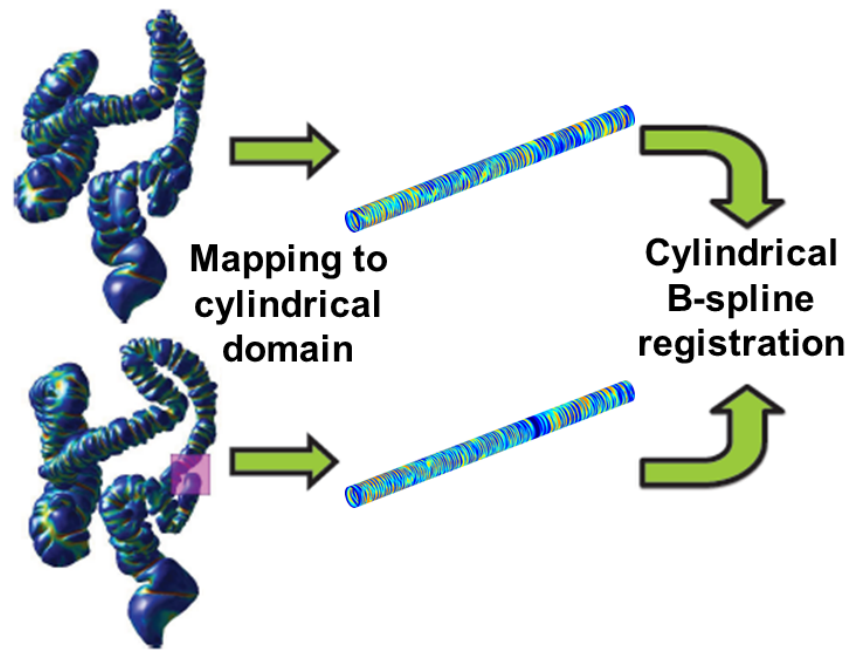

Fig. 1. Prone (top) to supine (bottom) registration of endoluminal colon surfaces in cylindrical space. The color coding indicates the local shape index $S I$ measurements which drive a non-rigid cylindrical B-spline registration - even in the case of local collapse (indicated by square).

\section{Methods and materials}

The external validation is based on publicly available CTC data [21]: the ACRIN CTC trial ${ }^{3}$ investigators have made 103 anonymized cases, from 15 US centers, with polyps (including reference standard) available on the National Cancer Institutes National Biomedical Imaging Archive (NBIA) ${ }^{4}$. These cases include

\footnotetext{
${ }^{3}$ http://www.acrin.org/TabID/151/Default.aspx

${ }^{4}$ https://imaging.nci.nih.gov/ncia/
} 
69 medium $(6-9 \mathrm{~mm})$ and 32 large $(>=10 \mathrm{~mm})$ polyps. These data represents cases that are generalizable to daily practice and were uninvolved with the development of the registration method. No attempt has been performed to select 'perfect' cases; cases were examined and excluded if: no independent CTC reference data were available (41), the polyp was not visible in both datasets (7), the dataset was incomplete/corrupted (3) or the cancer/polyp was $>30 \mathrm{~mm}$ (3). This leaves 49 cases with 66 polyps (38 large, 28 medium) used for validation. Each present polyp was identified by an experienced observer (DJB) using the independent reference data and prone and supine 3D polyp volumes were labeled manually using ITK-snap ${ }^{5}$. The segmental distribution of polyps in the validation sample $(\mathrm{n}=66)$ were compared to polyps $\geq 6 \mathrm{~mm}(\mathrm{n}=547)$ from the entire ACRIN CTC study (21) $(\mathrm{n}=2525)$ to investigate the likely generalizability of our results. By adopting the criteria proposed by Hara et al. [14], $55 \%$ of validation cases $(\mathrm{n}=27)$ had excess residual fluid compared to $52 \%$ (1313) of the total CT colonography studies from the same trial. $47 \%$ (23) had at least one region of complete luminal collapse, similar to the $48 \%$ (50) observed in the total, 103, positive cases in the publicly available database.

\section{Assessment of clinical utility}

Currently, standard clinical workstations for CT colonography interpretation allow the rendering of a virtual endoscopic or virtual fly-through views in order to replicate the view during colonoscopy. We can judge the usefulness of our proposed registration algorithm in clinical practice using these three-dimensional renderings. To score the registration result, a polyp conspicuity grading was developed by two experienced radiologists (DJB, SH): after successfully establishing the prone-to-supine correspondence over the full colon surface, the user is automatically guided to the corresponding view in opposing data set when clicking on a potential polyp (see Fig. 2). The endoluminal display is generated using a standard $120^{\circ}$ field-of-view camera model and centering the camera origin at the corresponding centerline position, pointing towards the surface registration result. The clinical usefulness is then judged by using the following objective polyp conspicuity score. The score is proportional to the amount of 'mousedriven navigation' necessary in order to bring the target polyp into view after registration:

- 5 'Successful': Polyp directly marked by registration prompt (Fig. 2)

- 4 'Successful': Polyp within same field of view (Fig. 3)

- 3 'Partially successful': Polyp becomes visible after $\pm 90^{\circ}$ pan

- 2 'Partially successful': Polyp becomes visible after $360^{\circ}$ pan

- 1 'Unsuccessful': Polyp only comes into view after navigation along centerline

Similarly, a 3D conspicuity assessment is performed in order to compare to NDACC. Here, we combine the scores of category 4 and 5 as NDACC will not

\footnotetext{
${ }^{5}$ www.itksnap.org
} 

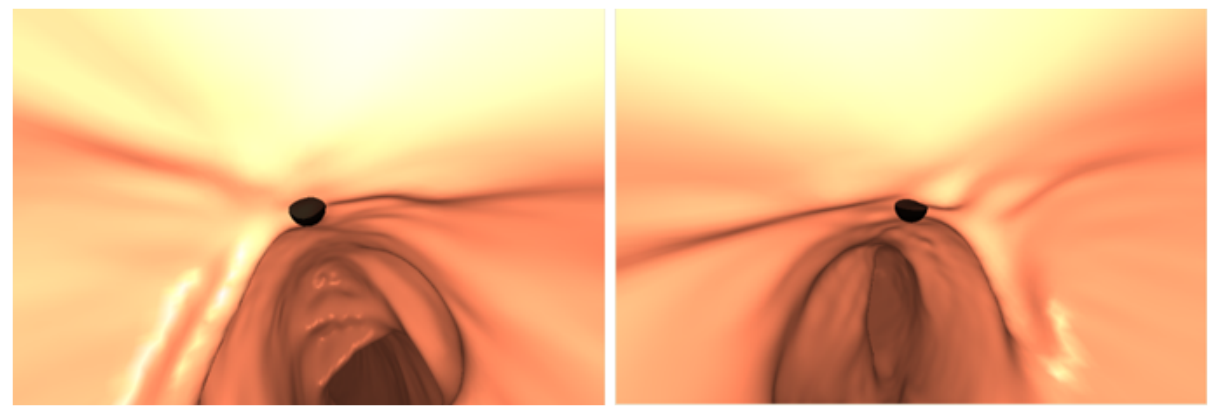

Fig. 2. Example of polyp conspicuity score of 5 ('direct hit'). The registration prompt (black dot) marks the polyp indicated by the observer in the supine dataset (left). Following registration, the algorithm centers the prone view to point towards the endoluminal coordinates calculated by the algorithm (right).
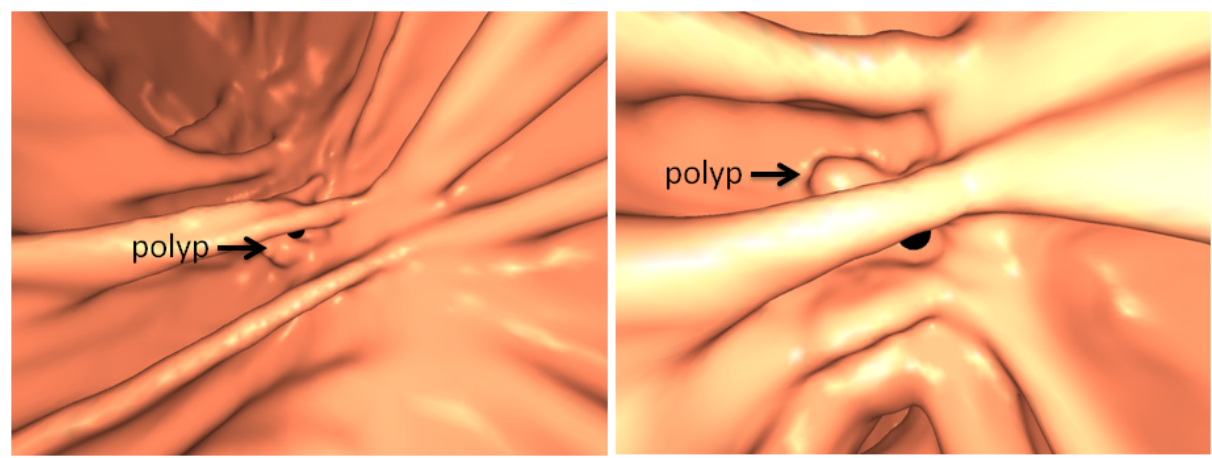

Fig. 3. Example of polyp conspicuity score of 4 ('near miss'). The registration prompt (black dot) marks the polyp indicated by the observer in the supine dataset (left). Following registration, the algorithm centers the prone 3D field of view to point towards the coordinates calculated by the algorithm (right). The algorithm fails to indicate the polyp (arrow) correctly but the polyp is clearly visible in the same field of view.

provide a registration prompt on the colon surface. It will only synchronize the virtual endoscopic views, looking straight along the centerline.

Furthermore, a conspicuity score was developed for aligning polyps using a standard multiplanar slice viewer for increasing necessity of mouse-driven navigation in all three orthogonal directions (axial, sagittal, coronal):

- 5 'Successful': Polyp directly marked by registration prompt.

- 4 'Successful': Polyp visible after $15 \mathrm{~mm}$ mouse-navigation

- 3 'Partially successful': Polyp visible after $\pm 20 \mathrm{~mm}$ mouse-navigation

- 2 'Partially successful': Polyp visible within $\pm 30 \mathrm{~mm}$ mouse-navigation

- 1 'Unsuccessful': Polyp not within $\pm 30 \mathrm{~mm}$ of registration prompt 
The polyp's apex was computed using the manual segmentations and was used to compute the $3 \mathrm{D}$ registration error and 2D slice displacement (measured in all 3 orthogonal directions). Both errors are reported in millimeters $(\mathrm{mm})$.

Polyps could be in the field of view or not depending on the particular local configuration of the endoluminal colon shape and centerline. In order to reduce any bias towards the direction of transformation, all polyps were transformed in prone-to-supine as well as in supine-to-prone directions. This resulted in a total of 132 point transformations of 66 polyps for the following analysis. A $5 \%$ significance level was a applied to all following cases when computing statistical significance. Despite the data reflecting clinical practice and depicting many areas of local colonic collapse, the registration was successfully computed in all of the cases $(100 \%)$.

\section{$5 \quad$ Results}

3D registration error The mean of the 3D registration error was $19.9 \mathrm{~mm}$ over the whole data set (66 polyps in 49 cases). This error measures the distance between the target polyp's apex and the point indicated on the colon surface by our proposed method. A median error of $11.9 \mathrm{~mm}$ was achieved and the $3 \mathrm{D}$ registration error ranged in the limits of $1.0 \mathrm{~mm}$ to $85.8 \mathrm{~mm}$. The $3 \mathrm{D}$ registration error did not vary significantly when comparing the polyp's location in different colonic segments $(\mathrm{p}=0.65)$. Furthermore, there was no significant increase in registration error within data cases exhibiting at least one local luminal collapse $(\mathrm{p}=0.075)$ using a Kruskal-Wallis test.

In order to provide a comparison to how a NDACC method would be used in clinical practice, the polyp's position along the centerline is found as the minimum distance between the polyps position on the colonic wall and all points along the centerline, following the approach by Wang et al. [5]. In comparison, using NDACC leads to a mean 3D error of $27.7 \mathrm{~mm}$ between apex of the polyp and the point indicated along the centerline. Here, our measured error is significantly smaller $(\mathrm{p}=0.001)$.

1D registration error Finding the polyp's position along the centerline further allows measuring the registration error as a 1D distance along centerline, summarized in Table 1. Using a non-parametric paired analysis (Wilcoxon Signed-Rank Test), our method gives no significant advantage over using NDACC when using all 66 polyps: $17.6 \mathrm{~mm}$ compared to $20.8 \mathrm{~mm}$ using NDACC. However, when comparing registration by colonic segment, the method is shows significant improvement over NDACC in the transverse, descending and sigmoid colon (see Table 1). A mean of $19.3 \mathrm{~mm}$ is achieved for these colonic segments in comparison $26.9 \mathrm{~mm}$ (NDACC). This is a significant improvement with $\mathrm{p}=0.047$. The largest improvement occurred in the transverse colon with a $15.3 \mathrm{~mm}$ reduction in registration error. This was anticipated as this segment was reported as being most mobile during positional change of the patient [2]. 
Table 1. 1D registration error, measured as displacement in along centerline [mm].

\begin{tabular}{lr|r|r}
\hline \hline $\begin{array}{l}\text { Colonic } \\
\text { segment }\end{array}$ & $\begin{array}{r}\text { Number of } \\
\text { polyps }\end{array}$ & $\begin{array}{r}\text { 1D error } \\
\text { proposed method }\end{array}$ & $\begin{array}{l}\text { 1D error } \\
\text { NDACC }\end{array}$ \\
\hline Rectum & 14 & 13.1 & 14.7 \\
Sigmoid & 15 & 20.9 & 23.6 \\
Descending & 11 & 18.1 & 27.5 \\
Transverse & 7 & 17.9 & 33.2 \\
Ascending & 12 & 22.7 & 17.2 \\
Cecum & 7 & 9.9 & 10.5 \\
\hline Total & 66 & 17.6 & 20.8 \\
\hline \hline
\end{tabular}

Polyp conspicuity score in 3D The registration achieved an direct alignment of the registration prompt in both opposing data sets in $89(67.4 \%)$ of cases using virtual fly-throughs (conspicuity score of 5; see Fig. 2). Of 132 point transformations, 20 (15.2\%) were transformed within the same field of view, leading to a score of 4 (see Fig. 3). Using the conspicuity grading, this leads to $82.7 \%$ 'successful' registrations. Nine (6.8\%) polyp transformations required up to 90 degrees mouse-driven rotation around the camera center in order to bring the polyp into view. In three accounts (2.3\%), a full 360 degrees of rotation were required to achieve the same. Therefore, a total of $9.1 \%$ were rated being aligned 'partially successful' for clinical relevance. A total of eleven (8.4\%) point transformations failed as further navigation along the centerline was necessary in order to bring the polyp into view. This compares favorable to using NDACC where a 'successful' (score 4) was only achieved in $37.1 \%$ of point transformations.

Polyp conspicuity score in 2D Using a purely multiplanar 2D slice 2D visualization, the registration error was assessed by judging utility expressed in necessary mouse-driven navigation in order to align the polyp apices in all three orthogonal directions. The algorithm correctly aligned the slices of $82(62.1 \%)$ pairs of prone and supine polyps within $\pm 15 \mathrm{~mm}$ of displacement and were therefore being judged 'successful' for 2D navigation. 28 (21.3\%) of cases were 'partially successful' registered, meaning that they were aligned within $\pm 30 \mathrm{~mm}$. A total of 22 registrations (16.7\%) failed as more than $\pm 30 \mathrm{~mm}$ of navigation was necessary in order to bring them into view. In comparison, NDACC achieved only $30(22.7 \%)$ 'successful' alignments within $\pm 15 \mathrm{~mm}$ of displacement. $78(59.1 \%)$ were judged 'partially successful' and $24(18.2 \%)$ failed and was not visible within $\pm 30 \mathrm{~mm}$ of navigation.

\section{Discussion}

In current clinical practice, prior to virtual colonoscopy, the interpreting clinician must check the validity of the workstation's proposed colonic segmentation, 
primarily to ensure the computed centerline takes the correct path to ensure correct navigation along the colonic lumen. Likewise, our registration algorithm currently relies on the user accepting the colon segmentation and the correct order of colonic segments, defining start and end points. Thereafter, the algorithm for establishing correspondence over the whole colonic surface is automated.

The assessment of registration accuracy used data from several hospitals unrelated to the development of the algorithm. This enables a generalizable assessment of the algorithm's performance which could be applied to normal clinical practice. Our study sample closely parallels the ACRIN data in quality of the bowel preparation and distension. Our algorithm compares favorably with previous methods which use optimally prepared cases or exclude cases exhibiting local collapses for validation. We provide full surface registration of the colonographic data that enables the indication of a specific location on the endoluminal surface. This would provide the observer with considerably more information, compared to a centerline position from where further navigation is necessary in order to find the target location. This is reflected in our choice of pre-specified criteria for polyp conspicuity using 3D endoscopic fly-through visualization following registration. Measuring the amount of navigation necessary in order to align prone and supine polyps after registration allows a direct comparison to centerline-based matching using NDACC. While it is intuitive that an accurate prone to supine registration would be beneficial for shortening interpretation time, further studies of clinical utility in everyday practice are necessary. For example, the effect on sensitivity and specificity when finding polyps needs to be evaluated.

This study is not without limitations: Some studies from the ACRIN validation archive were necessarily excluded due to inability of our radiologists to confirm the presence of a polyp in both the prone and supine acquisitions. It is therefore possible that some, inadequately distended studies were excluded from the analysis. Nevertheless, we postulate that the NDACC methods, due to its reliance on a continuous colonic segmentation, without regions of collapse, would have fared even worse that the proposed method when dealing with these data. In addition, while the processing of the endoluminal centerline occurs rapidly and is available to the reporting radiologist at the time of reporting, our method, at present, requires considerable processing time for surface extraction, feature matching and non-rigid registration. However, once this step has been complete, transferring between matched prone and supine locations is practically instantaneous. This is reminiscent of another, well-established adjunct to CT colonographic interpretation - computer-aided detection (CAD) which, although now integrated into many vendor platforms, initially required several hours of processing time. We anticipate that with the integration of multi-threading and/or GPU-technology we can reduce this such that integration into routine work-flow could be possible.

In summary, we successfully applied computer-assisted prone-supine registration of specific points (polyps) on the endoluminal surface. The used CT colonography data used for validation was from a subset of a larger multi-center 
trial. Resulting registration accuracies show promise of the ability to rapidly and automatically match switch between prone and supine positions and compare favorably to using NDACC. This will further facilitate the interpretation of CT colonography data.

\section{Acknowledgment}

The authors gratefully acknowledge financial support for this work from the NIHR program: Imaging diagnosis of colorectal cancer: Interventions for efficient and acceptable diagnosis in symptomatic and screening populations (Grant No. RP-PG-0407-10338) and the EPSRC-CRUK Comprehensive Cancer Imaging Centre of UCL and KCL (Grant No. C1519AO). Image data used in this research were obtained from The Cancer Imaging Archive ${ }^{6}$ sponsored by the Cancer Imaging Program, DCTD/NCI/NIH.

\section{References}

1. Yee, J., Kumar, N., Hung, R., Akerkar, G., Kumar, P., Wall, S.: Comparison of supine and prone scanning separately and in combination at ct colonography1. Radiology 226(3) (2003) 653-661 1

2. Punwani, S., Halligan, S., Tolan, D., Taylor, S., Hawkes, D.: Quantitative assessment of colonic movement between prone and supine patient positions during CT colonography. Br. J. Radiol. 82(978) (2009) 475-481 1, 5

3. Acar, B., Napel, S., Paik, D., Li, P., Yee, J., Beaulieu, C., Jeffrey, R.: Registration of supine and prone ct colonography data: Method and evaluation. Radiology 221(332) (2001) 3321

4. Li, P., Napel, S., Acar, B., Paik, D., Jeffrey Jr, R., Beaulieu, C.: Registration of central paths and colonic polyps between supine and prone scans in computed tomography colonography: Pilot study. Med. Phys. 31(10) (2004) 2912-2923 1

5. Wang, S., Yao, J., Liu, J., Petrick, N., Van Uitert, R., Periaswamy, S., Summers, R.: Registration of prone and supine CT colonography scans using correlation optimized warping and canonical correlation analysis. Med. Phys. 36(12) (2009) $5595-56031,5$

6. Näppi, J., Okamura, A., Frimmel, H., Dachman, A., Yoshida, H.: Region-based supine-prone correspondence for reduction of false-positive CAD polyp candidates in CT colonography. Acad. Radiol. 12 (2005) 695-707 1

7. de Vries, A., Truyen, R., Van der Peijl, J., Florie, J., van Gelder, R., Gerritsen, F., Stoker, J.: Feasibility of automated matching of supine and prone CT-colonography examinations. Br. J. Radiol. 79(945) (2006) 740-744 1

8. Summers, R., Swift, J., Dwyer, A., Choi, J., Pickhardt, P.: Normalized distance along the colon centerline: a method for correlating polyp location on ct colonography and optical colonoscopy. Am. J. Roentgenol. 193(5) (2009) 1296-1304 1

9. Suh, J.W., Wyatt, C.L.: Registration of prone and supine colons in the presence of topological changes. Proc. SPIE Med. Imaging 6916(1) (2008) 69160C 1

\footnotetext{
$\overline{{ }^{6} \text { http://cancerimagingarchive.net/ }}$
} 
10. Suh, J., Wyatt, C.: Deformable registration of supine and prone colons for computed tomographic colonography. J. Comput. Assist. Tomogr. 33(6) (2009) 9029111

11. Huang, A., Roy, D., Summers, R., Franaszek, M., Petrick, N., Choi, J., Pickhardt, P.: Teniae coli-based circumferential localization system for CT colonography: Feasibility study. Radiology 243(2) (2007) 551-560 1

12. Zeng, W., Marino, J., Chaitanya Gurijala, K., Gu, X., Kaufman, A.: Supine and prone colon registration using quasi-conformal mapping. IEEE Trans. Vis. Comput. Graph. 16(6) (2010) 1348-1357 1

13. Fukano, E., Oda, M., Kitasaka, T., Suenaga, Y., Takayama, T., Takabatake, H., Mori, M., Natori, H., Nawano, S., Mori, K.: Haustral fold registration in CT colonography and its application to registration of virtual stretched view of the colon. Proc. SPIE Med. Imaging 7624(1) (2010) 7624201

14. Hara, A., Kuo, M., Blevins, M., Chen, M., Yee, J., Dachman, A., Menias, C., Siewert, B., Cheema, J., Obregon, R., et al.: National ct colonography trial (acrin 6664): comparison of three full-laxative bowel preparations in more than 2500 average-risk patients. Am. J. Roentgenol. 196(5) (2011) 1076-1082 1, 3

15. Halligan, S., Taylor, S., Dehmeshki, J., Amin, H., Ye, X., Tsang, J., Roddie, M.: Computer-assisted detection for ct colonography: external validation. Clin. Radiol. 61(9) (2006) 758-763 1

16. Roth, H.R., McClelland, J.R., Boone, D.J., Modat, M., Cardoso, M.J., Hampshire, T.E., Hu, M., Punwani, S., Ourselin, S., Slabaugh, G.G., Halligan, S., Hawkes, D.J.: Registration of the endoluminal surfaces of the colon derived from prone and supine ct colonography. Medical Physics 38(6) (2011) 3077-3089 2

17. Hampshire, T., Roth, H., Hu, M., Boone, D., Slabaugh, G., Punwani, S., Halligan, S., Hawkes, D.: Automatic prone to supine haustral fold matching in CT colonography using a markov random field model. Medical Image Computing and Computer-Assisted Intervention-MICCAI 2011 (2011) 508-515 2

18. Jin, M., Kim, J., Luo, F., Gu, X.: Discrete surface Ricci flow. IEEE Trans. Vis. Comput. Graphics 14(5) (2008) 1030-1043 2

19. Modat, M., Ridgway, G., Taylor, Z., Lehmann, M., Barnes, J., Hawkes, D., Fox, N., Ourselin, S.: Fast free-form deformation using graphics processing units. Comput. Meth. Prog. Bio. 98(3) (2010) 278-284 2

20. Yoshida, H., Nappi, J.: Three-dimensional computer-aided diagnosis scheme for detection of colonic polyps. IEEE Trans. Med. Imaging 20(12) (2002) 1261-1274 2

21. Johnson, C., Chen, M., Toledano, A., Heiken, J., Dachman, A., Kuo, M., Menias, C., Siewert, B., Cheema, J., Obregon, R., et al.: Accuracy of CT colonography for detection of large adenomas and cancers. N. Engl. J. Med. 359(12) (2008) 1207-1217 3 